\title{
Backward-Forward Reachable Set Splitting for State-Constrained Differential Games
}

\author{
Xuhui Feng, Mario E. Villanueva, Boris Houska
}

\begin{abstract}
This paper is about a set-based computing method for solving a general class of two-player zero-sum Stackelberg differential games. We assume that the game is modeled by a set of coupled nonlinear differential equations, which can be influenced by the control inputs of the players. Here, each of the players has to satisfy their respective state and control constraints or loses the game. The main contribution is a backward-forward reachable set splitting scheme, which can be used to derive numerically tractable conservative approximations of such two player games. In detail, we introduce a novel class of differential inequalities that can be used to find convex outer approximations of these backward and forward reachable sets. This approach is worked out in detail for ellipsoidal set parameterizations. Our numerical examples illustrate not only the effectiveness of the approach, but also the subtle differences between standard robust optimal control problems and more general constrained two-player zero-sum Stackelberg differential games.
\end{abstract}

Keywords: optimal control, set-based computing, differential games

\section{Introduction}

The origins of differential games and game-theoretic optimal control go back to [22]. A historical overview of the early developments, roughly ranging from 1950-1970, can be found in 9]. There it becomes clear that, since their inception, the theories of differential games and optimal control have been deeply intertwined [30. The mathematical foundation of modern differential game theory, was established between 1970-1990. Precise definitions and a mature mathematical framework for this theory can, for example, be found in the books by [16] as well as 24].

The question of how to define an appropriate mathematical model for a game has, in general, no unique answer. This is due to the fact that the construction of such a model may depend on many different aspects. One of these aspects is the information each player has about other players, including their goals, their ability (and willingness) to communicate, and their willingness to agree on actions and rules [3, 5, 28. The focus of this paper is on a specific class of games, 
namely, two-player zero-sum Stackelberg games [7, 35], also known as "worstcase games". Here, Player 1 makes a decision and announces it to the other player, who has a conflicting objective. Stackelberg games arise in economics, marketing and policy making [14, but their applicability also extends to control systems [6, 29].

In a context of mathematical programming applied to static zero-sum Stackelberg games, one can distinguish between semi-infinite programming (SIP) [17] and generalized semi-infinite programming (GSIP) problems [23. Both classes of problems can be used to model zero-sum Stackelberg games, but in SIP the feasible set of the second player is assumed to be constant. This is in contrast to GSIP, where the decision of the first player affects the feasible set of the optimization problem of the second player. Notice that there exists a vast body of literature on numerical methods for both SIP and GSIP [31, 13.

The main difference between static and differential games is that, in the latter case, the state of the game is modeled by a differential equation [16],

$$
\forall t \in[0, T], \quad \dot{x}(t)=f\left(x(t), u_{1}(t), u_{2}(t)\right) .
$$

Here, the decision variables of the players are functions of time: Player 1 chooses the input function $u_{1}$, while Player 2 chooses $u_{2}$. A Stackelberg differential game can either be played in open-loop or closed-loop mode. In the former, Player 1 chooses $u_{1}$ first and announces his decision to Player 2. In contrast, in closedloop mode both players make their decisions simultaneously and continuously in time, for example, based on the current state $x(t)$ of the system.

Worst-case robust optimal control problems are a special class of zero-sum differential games, where the control $u_{2}$ is bounded by a given set. For example, $H_{\infty}$ control problems can be interpreted as differential games against nature [4]. In the mathematical control literature robust optimal control problems are frequently analyzed by means of Hamilton-Jacobi equations [2, 10. Modern numerical methods for robust optimal control, both in open- and closed-loop, are often based on set-theoretic considerations [8, 20, 26.

In order to understand the contributions of this paper, it is important to be aware of one fact: standard robust optimal control problems are differential games which do not enforce state constraints on the adverse player. This is in contrast to differential games played against a rational opponent, who might develop sophisticated strategies but agrees to act subject to the rules of the game. Formally, such rules can be modeled via state constraints $\mathbb{X}_{1}(t) \subseteq \mathbb{R}^{n_{x}}$ and $\mathbb{X}_{2}(t) \subseteq \mathbb{R}^{n_{x}}$. These sets are such that Player 1 loses if the constraint

$$
\forall t \in[0, T], \quad x(t) \in \mathbb{X}_{1}(t)
$$

is violated, while Player 2 loses if the constraint

$$
\forall t \in[0, T], \quad x(t) \in \mathbb{X}_{2}(t)
$$

is violated. For example, in a football game an offside can be modeled by a suitable state constraint set $\mathbb{X}_{2}(t)$ that is related to the relative positions (differential states) of the players and the ball. Rational players could, in principle, 
exploit their explicit knowledge of the state constraints of their opponents. In football, for example, one team could let the adverse team run into an offside trap. To draw an analogy to static games, one could state that zero-sum Stackelberg differential games with state constraints are to robust optimal control problems what GSIP problems are to SIP problems. In the context of general differential games, the actions of the first player affect the set of feasible actions of the second player. This is because the solution of the differential equation depends on both $u_{1}$ and $u_{2}$.

Compared to the vast amount of literature on numerical methods for standard robust optimal control, the number of articles on zero-sum differential games with state constraints on both players is rather limited. An interesting historical example for a differential game with state constraints is the famous "man and lion" problem. In this game, both players have equal maximum speed, and are both constrained to stay in a circular arena. The rather surprising fact that the man can survive infinitely long without being caught by the lion has been proven in 1952 by Besicovitch; [27, see]. Most contributions in the area of state-constrained zero-sum differential games use, in one way or another, concepts from viability theory [1. For example, 11] used viability kernel techniques to construct numerical methods for differential games with separable dynamics and state constraints. An overview of recent advances in zero-sum differential games with state constraints can also be found in [12.

The main contribution of this paper is a set-based computing framework for analyzing and constructing approximate, yet conservative solutions of zero-sum Stackelberg differential games. The proposed framework is able to deal with coupled dynamics as well as state constraints for both players. The set-based problem formulation is outlined in Section 2. In contrast to [11, we do not assume that the dynamics of the game are separable. Section 3 introduces a generic backward-forward reachable set splitting result for such non-separable two player differential games, which is presented in Theorem 1. This result is then used to construct convex outer approximations of the constrained reachable set of the second player via a system of generalized differential inequalities, as summarized in Theorem 2. Theorem 3 specializes this result for ellipsoidal set parameterizations. In Section 4, the latter construction is leveraged in order to construct a standard optimal control problem with boundary constraints, whose solution conservatively approximates the solution of the original set-based problem. This problem can be solved using existing, gradient-based, optimal control algorithms. The developments of this paper are demonstrated through a numerical example implemented using the optimal control software ACADO Toolkit [19, in Section 4.1. Section 5 concludes the paper.

Notation The set of $n$-dimensional $L_{1}$-integrable functions is denoted by $\mathbb{L}_{1}^{n}$ while $\mathbb{W}_{1,1}^{n}$ denotes the associated Sobolev space of weakly differentiable functions with $L_{1}$ integrable derivatives. The set of compact and convex compact subsets of $\mathbb{R}^{n}$ are denoted by $\mathbb{K}^{n}$ and $\mathbb{K}_{C}^{n}$, respectively. For a set $Z \subseteq \mathbb{R}^{n}$, $\mathcal{P}(Z) \subseteq \mathbb{R}^{n}$ denotes its power set, which includes the empty set, denoted by $\varnothing$. 
Moreover, $\operatorname{int}(Z)$ denotes the interior of a set $Z \subseteq \mathbb{R}^{n}$ and $\operatorname{cl}(Z)$ its closure in $\mathbb{R}^{n}$. The support function of a set $Z$ is defined as

$$
\forall c \in \mathbb{R}^{n}, \quad V[Z](c)=\sup _{z \in Z} c^{\top} z .
$$

Additionally, we define $V[\varnothing](c)=-\infty$. The sets of positive semidefinite and positive definite $n$-dimensional matrices is denoted by $\mathbb{S}_{+}^{n}$ and $\mathbb{S}_{++}^{n}$. An ellipsoid with center $q \in \mathbb{R}^{n}$ and shape matrix $Q \in \mathbb{S}_{+}^{n}$ is given by

$$
\mathcal{E}(q, Q)=\left\{q+Q^{\frac{1}{2}} v \mid \exists v \in \mathbb{R}^{n}: v^{\top} v \leq 1\right\},
$$

where $Q^{\frac{1}{2}}$ can be any square root of $Q$, as the unit disc in $\mathbb{R}^{n}$ remains invariant under orthogonal transformations.

\section{Open-loop Stackelberg differential games}

This paper is about two-player differential games whose state, $x \in \mathbb{W}_{1,1}^{n_{x}}$, satisfies a differential equation of the form

$$
\begin{array}{ll}
\forall t \in[0, T], & \dot{x}(t)=f\left(x(t), u_{1}(t), u_{2}(t)\right), \\
\text { with } & x(0)=x_{0} .
\end{array}
$$

Here, Player 1 chooses the control input $u_{1}:[0, T] \rightarrow \mathbb{U}_{1}$ while Player 2 chooses the control input $u_{2}:[0, T] \rightarrow \mathbb{U}_{2}$.

Assumption 1 The right-hand side function, $f$, is jointly continuous in $x, u_{1}, u_{2}$ and locally Lipschitz continuous in $x$.

Assumption 2 The control constraint sets $\mathbb{U}_{1}, \mathbb{U}_{2} \subseteq \mathbb{R}^{n_{u}}$ are non-empty, convex, and compact.

For simplicity of presentation, it is also assumed that the initial state $x_{0} \in \mathbb{R}^{n_{x}}$ is a given constant. The developments in this paper can easily be generalized for the case that the initial value is chosen by one of the players or to the case where the inputs have different dimensions.

Remark 1 Two-player differential games can sometimes be represented using a differential equation system of the form

$$
\begin{array}{ll}
\forall t \in[0, T], & \dot{x}_{1}(t)=f_{1}\left(x_{1}(t), u_{1}(t)\right), \\
\forall t \in[0, T], & \dot{x}_{2}(t)=f_{2}\left(x_{2}(t), u_{2}(t)\right), \\
\text { with } & x(0)=x_{0} .
\end{array}
$$

These systems are called separable, since each function $f_{i}, i \in\{1,2\}$, depends only on the state $x_{i}$ and control $u_{i}$. As an example, consider a simple twocar race: the states of each car (position, orientation, and velocities) are only 
functions of its own controls (acceleration and steering). Likewise, there are systems that cannot be formulated as (2) easily. Consider for example two children on a seesaw: each child may shift its own weight independently (control input), while the state of the system (position and velocity of the seesaw) is simultaneously affected by both control inputs. We keep the formulation general, as every separable system can be written as (1) by introducing the stacked state $x^{\boldsymbol{\top}}=\left(x_{1}^{\boldsymbol{\top}}, x_{2}^{\boldsymbol{\top}}\right)$.

\subsection{State constraints}

State constraints can be used to define the rules of a game. Here, we consider two given - and potentially time varying - state constraint sets, $\mathbb{X}_{1}(t), \mathbb{X}_{2}(t) \subseteq \mathbb{R}^{n_{x}}$. Player 1 loses if the constraint

$$
\forall t \in[0, T], \quad x(t) \in \mathbb{X}_{1}(t)
$$

is violated. Likewise, Player 2 loses if the constraint

$$
\forall t \in[0, T], \quad x(t) \in \mathbb{X}_{2}(t)
$$

is violated. Depending on the particular definitions of $\mathbb{X}_{1}$ and $\mathbb{X}_{2}$, there may be situations in which both players lose, one of the players loses, or no one loses.

Remark 2 There are games, where the state constraint sets $\mathbb{X}_{1}$ and $\mathbb{X}_{2}$ coincide. Consider again the two-car race from Remark 1: one may be interested in enforcing a collision avoidance constraint. This gives rise to a coupled state constraint involving the positions of both cars. If a collision occurs, both cars are out of the race. Thus, if there are no further constraints, we have $\mathbb{X}_{1}=\mathbb{X}_{2}$. However, as soon as we introduce the additional rule that Player 1 loses if the first car leaves the road while Player 2 loses if the second car is not staying on track, we have $\mathbb{X}_{1} \neq \mathbb{X}_{2}$ (in this example, Player 1 does not necessary lose if the second player's car is not staying on the road and vice-versa).

\section{$2.2 \quad$ Feasibility}

We use the symbol $\mathbb{X}\left[u_{1}\right]$ to denote the set of feasible state trajectories that the second player can realize,

$$
\mathbb{X}\left[u_{1}\right]=\left\{\begin{array}{l|l}
x \in \mathbb{W}_{1,1}^{n_{x}} & \begin{array}{l}
\exists u_{2} \in \mathbb{L}_{1}^{n_{u}}: \forall \tau \in[0, T] \\
\dot{x}(\tau)=f\left(x(\tau), u_{1}(\tau), u_{2}(\tau)\right), \\
x(\tau) \in \mathbb{X}_{2}(\tau), u_{2}(\tau) \in \mathbb{U}_{2}, \\
x(0)=x_{0}
\end{array}
\end{array}\right\}
$$

Thus, the set-valued function $X\left[u_{1}\right]: \mathbb{R} \rightarrow \mathcal{P}\left(\mathbb{R}^{n_{x}}\right)$, given by

$$
\forall t \in[0, T], \quad X\left[u_{1}\right](t)=\left\{x(t) \in \mathbb{R}^{n_{x}} \mid x \in \mathbb{X}\left[u_{1}\right]\right\},
$$

denotes the reachable set in the state space. 
Definition 1 A control input $u_{1} \in \mathbb{L}_{1}^{n_{u}}$ with $u_{1}:[0, T] \rightarrow \mathbb{U}_{1}$ is called lowerlevel feasible if

$$
\mathbb{X}\left[u_{1}\right] \neq \varnothing .
$$

Otherwise, $u_{1}$ is called lower-level infeasible.

Notice that if Player 1 chooses a control input $u_{1}$ that is lower-level infeasible, Player 2 is forced to violate the rules of the game. Because this paper focuses on games in which such behavior of Player 1 is unwanted, we introduce the following definition of upper level feasibility.

Definition 2 A control input $u_{1} \in \mathbb{L}_{1}^{n_{u}}$ with $u_{1}:[0, T] \rightarrow \mathbb{U}_{1}$ is called upperlevel feasible if it is lower-level feasible and

$$
\forall t \in[0, T], \quad X\left[u_{1}\right](t) \subseteq \mathbb{X}_{1}(t) .
$$

Otherwise, $u_{1}$ is called upper-level infeasible.

Remark 3 Enforcing the constraint $\mathbb{X}\left[u_{1}\right] \neq \varnothing$ is equivalent to introducing the rule that Player 1 loses the game if $u_{1}$ is lower-level infeasible. At this point, one should be clear in mind that requiring lower-level feasibility does not imply that Player 1 is not allowed to "win" the game. The conditions for lower-and upper-level feasibility merely define under which conditions Player 1 loses the game.

Remark 4 Robust optimal control [21] considers games with $\mathbb{X}_{2}=\mathbb{R}^{n_{x}}$ with the disturbances being the input of the adverse player. In such case, all inputs $u_{1}$ are lower-level feasible, since the second player has no state constraints that could possibly become infeasible.

\subsection{Constrained open-loop zero-sum differential games}

The goal of this paper is to analyze and approximately solve constrained openloop zero-sum differential games with Stackelberg information structure. Thus, we assume that Player 1 chooses a strategy and announces it to Player 2. An optimal open loop strategy for Player 1 is any solution of

$$
\begin{aligned}
& \inf _{u_{1}} \mathcal{M}\left(X\left[u_{1}\right](T)\right) \\
& \text { s.t. } \begin{cases}\mathbb{X}\left[u_{1}\right] \quad \neq \varnothing \\
X\left[u_{1}\right](t) \subseteq \mathbb{X}_{1}(t) \text { for all } t \in[0, T] \\
u_{1}(t) \quad \in \mathbb{U}_{1} & \text { for all } t \in[0, T] .\end{cases}
\end{aligned}
$$

We assume that a Mayer term $m: \mathbb{R}^{n_{x}} \rightarrow \mathbb{R}$ is given and that

$$
\mathcal{M}(X)=\sup _{\xi \in X} m(\xi)
$$

denotes the supremum (worst-case value) of $m$ on a given set $X$. Notice that (6) is feasible if and only if there exists a control input $u_{1}$ that is upper-level feasible. Additionally, we recall that if $u_{1}$ is a feasible point of (6), then $u_{1}$ is-by construction of the rules of our game - also lower-level feasible. 
Remark 5 If there exists an equilibrium solution $\left(u_{1}^{\star}, u_{2}^{\star}\right)$ (min-max point) of the open-loop Stackelberg zero-sum differential game, it can be computed by first finding a minimizer $u_{1}^{\star}$ of (6). In this case, $u_{2}^{\star}$ must be a maximizer of

$$
\max _{x, u_{2}} m(x(T)) \text { s.t. }\left\{\begin{array}{l}
\forall t \in[0, T]: \\
\dot{x}(t)=f\left(x(t), u_{1}^{\star}(t), u_{2}(t)\right) \\
x(t) \in \mathbb{X}, u_{2}(t) \in \mathbb{U}_{2} \\
x(0)=x_{0} .
\end{array}\right.
$$

Throughout this paper it is assumed that the decision of whether one of the players has won the game is made a posteriori, after both players have played their strategies. This final decision is based on the objective value $m(x(T))$, where $x(T)$ denotes the state of the system at time $T$. As we can always add constant offsets to $m$, we say that Player 1 wins the game if $u_{1}$ is upper-level feasible and $m(x(T)) \leq 0$. Similarly, Player 2 wins if $x(t) \in \mathbb{X}_{2}(t)$ for all $t \in[0, T]$ and $m(x(T))>0$. Notice that this definition is consistent in the sense that at most one player can win the game and it is impossible that one of the players wins and loses a game simultaneously.

Remark 6 One can also consider closed-loop games whose dynamics are given by

$$
\begin{array}{ll}
\forall t \in[0, T], & \dot{x}(t)=F\left(x(t), \mu_{1}(t, x(t)), \mu_{2}(t, x(t))\right. \\
\text { with } & x(0)=x_{0} .
\end{array}
$$

Here, Player 1 chooses the feedback law $\mu_{1}: \mathbb{R} \times \mathbb{R}^{n_{x}} \rightarrow \mathbb{U}_{1}$ while Player 2 chooses the feedback law $\mu_{2}: \mathbb{R} \times \mathbb{R}^{n_{x}} \rightarrow \mathbb{U}_{2}$. Closed-loop games in full generality are significantly harder to solve and analyze than open-loop games, even without the presence of state-constraints. Nevertheless, by restricting the search to parametric feedback laws, for example, affine feedback laws of the form

$$
\mu_{1}(x)=K_{1}(t) x+k_{1}(t) \quad \text { and } \quad \mu_{2}(x)=K_{2}(t) x+k_{2}(t),
$$

one can formulate closed-loop games in the form of (6) by regarding the control law coefficients $u_{1}=\left(\operatorname{vec}\left(K_{1}\right)^{\top}, k_{1}^{\top}\right)^{\top}$ and $u_{2}=\left(\operatorname{vec}\left(K_{2}\right)^{\top}, k_{2}^{\top}\right)^{\top}$ as the inputs of the first and second player, respectively.

\section{Backward-forward reachable set splitting}

The goal of this section is to analyze the reachable sets $X\left[u_{1}\right](t)$. Notice that, due to the presence of state constraints for Player 2, it is non-trivial to ensure lower-level feasibility of an input $u_{1}$ (see Remark 4). In particular, the state constraints $\mathbb{X}_{2}(\cdot)$ induce a coupling in time; that is, knowing the reachable state at time $t$, requires knowledge of the state trajectories on the whole time horizon. 
In order to remove the coupling in time, we introduce the set-valued function $X_{\mathrm{B}}\left[u_{1}\right]: \mathbb{R} \rightarrow \mathcal{P}\left(\mathbb{R}^{n_{x}}\right)$ given by

$$
X_{\mathrm{B}}\left[u_{1}\right](t)=\left\{\begin{array}{l|l}
\xi \in \mathbb{R}^{n_{x}} & \begin{array}{l}
\exists x \in \mathbb{W}_{1,1}^{n_{x}}, \exists u_{2} \in \mathbb{L}_{1}^{n_{u}}: \\
\forall \tau \in[t, T], \\
\dot{x}(\tau)=f\left(x(\tau), u_{1}(\tau), u_{2}(\tau)\right) \\
x(\tau) \in \mathbb{X}_{2}(t), u_{2}(\tau) \in \mathbb{U}_{2}, \\
x(t)=\xi
\end{array}
\end{array}\right\} .
$$

The set $X_{\mathrm{B}}\left[u_{1}\right](t)$ can be interpreted as the set of all states $x(t)$ of the game at time $t$ for which the second player is able to satisfy the rules of the game on the remaining time interval $[t, T]$. Next, we introduce the set-valued function $X_{\mathrm{F}}\left[u_{1}\right]: \mathbb{R} \rightarrow \mathcal{P}\left(\mathbb{R}^{n_{x}}\right)$ given by

$$
X_{\mathrm{F}}\left[u_{1}\right](t)=\left\{\begin{array}{l|l}
\xi \in \mathbb{R}^{n_{x}} & \begin{array}{l}
\exists x \in \mathbb{W}_{1,1}^{n_{x}}, \exists u_{2} \in \mathbb{L}^{n_{u}}: \\
\forall \tau \in[0, t] \\
\dot{x}(\tau)=f\left(x(\tau), u_{1}(\tau), u_{2}(\tau)\right) \\
x(\tau) \in X_{\mathrm{B}}\left[u_{1}\right](\tau), u_{2}(\tau) \in \mathbb{U}_{2} \\
x(0)=x_{0}, x(t)=\xi
\end{array}
\end{array}\right\}
$$

The set-valued function $X_{\mathrm{F}}\left[u_{1}\right](t)$ is called the constrained forward reachable set of the system at time $t$. The following theorem establishes the fact that $X$ and $X_{\mathrm{F}}$ coincide.

Theorem 1 The equation $X\left[u_{1}\right]=X_{\mathrm{F}}\left[u_{1}\right]$ holds for all input functions $u_{1} \in$ $\mathbb{L}_{1}^{n_{u}}$.

Proof. Let $t \in[0, T]$ and $u_{1} \in \mathbb{L}_{1}^{n_{u}}$ be given. The goal of the first part of this proof is to establish the inclusion

$$
X_{\mathrm{F}}\left[u_{1}\right](t) \subseteq X\left[u_{1}\right](t) .
$$

Let the functions $x_{\mathrm{F}} \in \mathbb{W}_{1,1}^{n_{x}}$ and $u_{2, \mathrm{~F}}:[0, t] \rightarrow \mathbb{U}_{2}$ be such that

$$
\begin{aligned}
& \dot{x}_{\mathrm{F}}(\tau)=f\left(x_{\mathrm{F}}(\tau), u_{1}(\tau), u_{2, \mathrm{~F}}(\tau)\right) \\
& x_{\mathrm{F}}(\tau) \in X_{\mathrm{B}}\left[u_{1}\right](\tau), \\
& x_{\mathrm{F}}(0)=x_{0}
\end{aligned}
$$

for all $\tau \in[0, t]$. Now, the definition of $X_{\mathrm{B}}\left[u_{1}\right]$ and $(11)$ imply that there exists a $x_{\mathrm{B}} \in \mathbb{W}_{1,1}^{n_{x}}$ and $u_{2, \mathrm{~B}}:[t, T] \rightarrow \mathbb{U}_{2}$ such that

$$
\begin{aligned}
\dot{x}_{\mathrm{B}}(\tau) & =f\left(x_{\mathrm{B}}(\tau), u_{1}(\tau), u_{2, \mathrm{~B}}(\tau)\right) \\
x_{\mathrm{B}}(\tau) & \in \mathbb{X}_{2}(\tau) \\
x_{\mathrm{B}}(t) & =x_{\mathrm{F}}(t)
\end{aligned}
$$


for all $\tau \in[t, T]$. Thus, we can construct the functions

$$
x(\tau)= \begin{cases}x_{\mathrm{F}}(\tau) & \text { if } 0 \leq \tau \leq t \\ x_{\mathrm{B}}(\tau) & \text { if } t \leq \tau \leq T\end{cases}
$$

and

$$
u_{2}(\tau)= \begin{cases}u_{2, \mathrm{~F}}(\tau) & \text { if } 0 \leq \tau \leq t \\ u_{2, \mathrm{~B}}(\tau) & \text { if } t \leq \tau \leq T\end{cases}
$$

with $u(\tau) \in \mathbb{U}_{2}$ for all $\tau \in[0, T]$. These functions satisfy

$$
\begin{array}{ll}
\dot{x}(\tau) & \left.10,13,15(x), u_{1}(\tau), u_{2}(\tau)\right) \\
x(\tau) & \mathbb{X}_{2}(\tau) \\
x(0) & x_{0}
\end{array}
$$

Inclusion (17) follows from (11) and 14 since, by construction, $X_{\mathrm{B}}$ satisfies $X_{\mathrm{B}}\left[u_{1}\right](\tau) \subseteq \mathbb{X}_{2}$ for all $\tau \in[0, t]$. Now, (16)-(18) imply that $x \in \mathbb{X}\left[u_{1}\right]$ and, consequently, $x(t) \in X\left[u_{1}\right](t)$. Thus, we have established (9).

For the second part of the proof, we need to show that the inclusion

$$
X\left[u_{1}\right](t) \subseteq X_{\mathrm{F}}\left[u_{1}\right](t)
$$

holds. Let $x \in \mathbb{X}\left[u_{1}\right]$ and $u_{2}$ satisfy

$$
\begin{aligned}
& \dot{x}(\tau)=f\left(x(\tau), u_{1}(\tau), u_{2}(\tau)\right) \\
& x(\tau) \in \mathbb{X}_{2} \\
& x(0)=0 .
\end{aligned}
$$

It is clear that $x$ satisfies the constraints in (7) on $[t, T], x(t) \in X_{\mathrm{B}}\left[u_{1}\right](t)$. But then, $x$ also satisfies the constraints in (8) on $[0, t]$, which implies $[19]$. Finally, 9 and $(19)$ yield the statement of the theorem.

\subsection{Construction of convex enclosures using generalized differential inequalities}

This section is concerned with the construction of enclosures for the reachability tube $X_{\mathrm{F}}\left[u_{1}\right]$.

Definition 3 Let $Z: \mathbb{R} \rightarrow \mathcal{P}\left(\mathbb{R}^{n}\right)$ be a set-valued function. A set-valued function $Y: \mathbb{R} \rightarrow \mathbb{K}_{\mathrm{C}}^{n}$ is called an enclosure of $Z$ on $[0, T]$ if $Y(t) \supseteq Z(t)$ for all $t \in[0, T]$. 
In the following, we use the shorthand

$$
\Gamma\left(\nu_{1}, c, Y, Z\right)=\left\{\begin{array}{l|l}
f\left(\xi, \nu_{1}, \nu_{2}\right) & \begin{array}{c}
c^{\top} \xi=V[Y](c) \\
\xi \in Y \cap \operatorname{int}(Z) \\
\nu_{2} \in \mathbb{U}_{2}
\end{array}
\end{array}\right\},
$$

which is defined for all $Z \in \mathbb{K}_{\mathrm{C}}^{n_{x}}, c \in \mathbb{R}^{n_{x}}$, and $\nu_{1} \in \mathbb{R}^{n_{u}}$.

The next theorem provides a basis for the construction of convex enclosures of $X_{\mathrm{F}}\left[u_{1}\right]$. It exploits the reach-set splitting structure of the backward-forward propagation scheme that has been introduced in Section 3 .

Theorem 2 Let Assumptions 1 and 2 be satisfied and let the Lebesgue integrable function $u_{1}: \mathbb{R} \rightarrow \mathbb{U}_{2}$ be given. Let $\mathbb{X}_{2}, Y_{\mathrm{B}}, Y_{\mathrm{F}}:[0, T] \rightarrow \mathbb{K}_{\mathrm{C}}^{n_{x}}$ be compact setvalued functions such that $V\left[Y_{\mathrm{B}}(\cdot)\right](c)$ and $V\left[Y_{\mathrm{F}}(\cdot)\right](c)$ are, for all $c \in \mathbb{R}^{n_{x}}$, Lipschitz continuous on $[0, T)$. If

$$
Y_{\mathrm{B}}(t) \cap \operatorname{int}\left(\mathbb{X}_{2}(t)\right) \neq \varnothing \quad \text { and } \quad Y_{\mathrm{F}}(t) \cap \operatorname{int}\left(Y_{\mathrm{B}}(t)\right)=\varnothing
$$

for all $t \in[0, T]$ and if the inequalities

$$
\begin{aligned}
\dot{V}\left[Y_{\mathrm{B}}(t)\right](c) & \leq-V\left[-\Gamma\left(u_{1}(t), c, Y_{\mathrm{B}}(t), \mathbb{X}_{2}(t)\right)\right](c) \\
\dot{V}\left[Y_{\mathrm{F}}(t)\right](c) & \geq V\left[\Gamma\left(u_{1}(t), c, Y_{\mathrm{F}}(t), Y_{\mathrm{B}}(t)\right)\right](c) \\
V\left[Y_{\mathrm{F}}(0)\right](c) & \geq c^{\top} x_{0} \\
V\left[Y_{\mathrm{B}}(T)\right](c) & \geq V\left[\mathbb{X}_{2}(T)\right](c)
\end{aligned}
$$

hold for all $t \in[0, T)$ and all $c \in \mathbb{R}^{n_{x}}$, then the set-valued function $Y_{\mathrm{F} \cap \mathrm{B}}: \mathbb{R} \rightarrow$ $\mathbb{K}_{\mathrm{C}}^{n_{x}}$ given by

$$
\forall t \in[0, T], \quad Y_{\mathrm{F} \cap \mathrm{B}}(t)=Y_{\mathrm{F}}(t) \cap Y_{\mathrm{F}}(t)
$$

is an enclosure of $X\left[u_{1}\right]$ on $[0, T]$.

Figure 1 visualizes the conditions of Theorem 2, The left panel shows two dimensional enclosures $Y_{\mathrm{F}}(t)$ and $Y_{\mathrm{B}}(t)$ in light and dark gray, respectively. Notice that the conditions in Theorem 2 are verified pointwise-in-time at the boundary of the enclosures. The black arrow indicates a given direction $c$ and the red line corresponds to the set of points of the associated supporting facet,

$$
F\left[Y_{\mathrm{F}}(t)\right](c)=\left\{\begin{array}{l|l}
\xi & \begin{array}{c}
c^{\top} \xi=V\left[Y_{\mathrm{F}}(t)\right](c) \\
\xi \in Y_{\mathrm{F}}(t)
\end{array}
\end{array}\right\},
$$

that are also in $\mathrm{Y}_{\mathrm{B}}(\mathrm{t})$. For a given control $u_{1}$ and starting from $F\left[Y_{\mathrm{F}}(t)\right](c) \cap$ $\operatorname{int}\left(Y_{\mathrm{B}}(t)\right)$, we have that any trajectory $x$ must satisfy

$$
\dot{x}(t) \in \bigcup_{\xi \in F\left[Y_{\mathrm{B}}(t)\right](c) \cap \operatorname{int}\left(Y_{\mathrm{B}}(t)\right)}\left\{f\left(\xi, u_{1}(t), \nu_{2}\right) \mid \nu_{2} \in \mathbb{U}_{2}\right\} .
$$

At this point, it is easy to see that the right-hand side of the above inclusion is exactly $\Gamma\left(u_{1}(t), c, Y_{\mathrm{F}}(t), Y_{\mathrm{B}}(t)\right)$-which is shown in the right panel, in light 

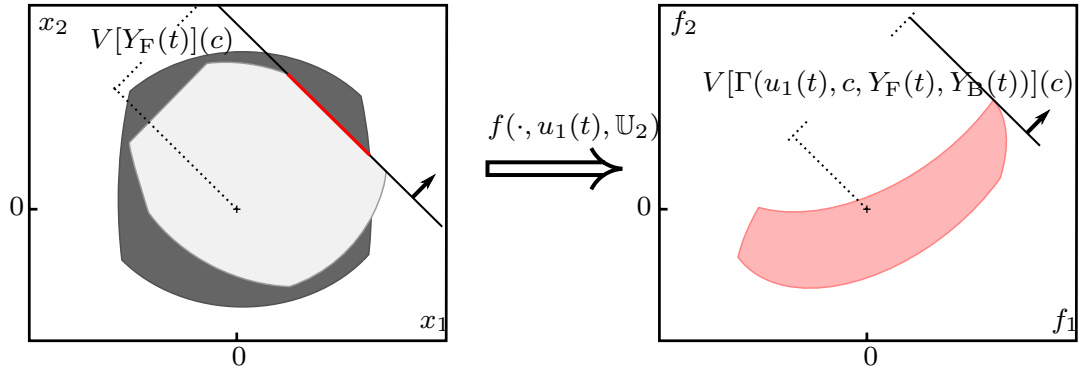

Figure 1: A sketch of the conditions in Theorem 2. Left: The sets $Y_{\mathrm{F}}(t)$ and $Y_{\mathrm{B}}(t)$ are shown in light and dark gray. The set $\{\xi \in$ $\left.Y_{\mathrm{F}}(t) \mid c^{\top} \xi=V\left[Y_{\mathrm{F}}(t)\right](c), \xi \in \operatorname{int}\left(Y_{\mathrm{B}}(t)\right)\right\}$, is shown in red. Right: The set $\Gamma\left(u_{1}(t), c, Y_{\mathrm{F}}(t), Y_{\mathrm{B}}(t)\right)$ is shown in light red. In both plots, the black arrow is the direction vector $c$. We also use the shorthand $f\left(\xi, u_{1}(t), \mathbb{U}_{2}\right)=$ $\left\{f\left(\xi, u_{1}(t), \nu_{2}\right) \mid \nu_{2} \in \mathbb{U}_{2}\right\}$.

red. Then, taking the support function of $\Gamma\left(u_{1}(t), c, Y_{\mathrm{F}}(t), Y_{\mathrm{B}}(t)\right)$ as a bound on $\dot{V}\left[Y_{\mathrm{F}}(t)\right](c)$ we are bounding the dynamics of the points on $F\left[Y_{\mathrm{F}}(t)\right](c) \cap$ $\operatorname{int}\left(Y_{\mathrm{B}}(t)\right)$.

A proof of Theorem 2 can be found in Appendix A.

\subsection{Ellipsoidal-valued enclosures for reachability tubes}

This section presents a practical construction of ellipsoidal enclosures based on Theorem 2. Our focus is on ellipsoidal set parameterizations of the form

$$
Y_{\mathrm{B}}(t)=\mathcal{E}\left(q_{\mathrm{B}}(t), Q_{\mathrm{B}}(t)\right) \quad \text { and } \quad Y_{\mathrm{F}}(t)=\mathcal{E}\left(q_{\mathrm{F}}(t), Q_{\mathrm{F}}(t)\right) .
$$

In particular, our goal is to develop a computational method for constructing the functions $q_{\mathrm{B}}, q_{\mathrm{F}}: \mathbb{R} \rightarrow \mathbb{R}^{n_{x}}$ as well as $Q_{\mathrm{B}}, Q_{\mathrm{F}}: \mathbb{R} \rightarrow \mathbb{S}_{++}^{n_{x}}$ in such a way that $Y_{\mathrm{B}}$ and $Y_{\mathrm{F}}$ satisfy the conditions from Theorem 2 .

Assumption 3 The sets $\mathbb{X}_{2}(t)$ are bounded for all $t \in[0, T]$.

Let $s: \mathbb{R} \rightarrow \mathbb{R}^{n_{x}}, S: \mathbb{R} \rightarrow \mathbb{S}_{++}^{n_{x}}$, and $(v, V) \in \mathbb{R}^{n_{u}} \times \mathbb{S}_{++}^{n_{u}}$ be given such that

$$
\mathbb{U}_{2} \subseteq \mathcal{E}(v, V) \quad \text { and } \quad \mathbb{X}_{2}(t) \subseteq \mathcal{E}(s(t), S(t))
$$

The existence of $s, S, v$ and $V$ is guaranteed, if Assumptions 2 and 3 hold. Moreover, let

$$
\Omega: \mathbb{R}^{n_{x} \times n_{x}} \times \mathbb{R}^{n_{x} \times n_{u}} \times \mathbb{R}^{n_{x}} \times \mathbb{R}^{n_{u}} \times \mathbb{R}^{n_{u}} \times \mathbb{S}_{+}^{n_{x}} \rightarrow \mathbb{S}_{+}^{n_{x}}
$$

be a nonlinearity bound such that

$$
f\left(x, u_{1}, u_{2}\right)-A(x-q)-B\left(u_{2}-v\right) \in \mathcal{E}\left(0, \Omega\left(A, B, q, u_{1}, v, Q\right)\right)
$$


is satisfied for all vectors $x \in \mathcal{E}(q, Q)$; all vectors $u_{1}, u_{2}, v$, and $q$; and all matrices $A, B$, and $Q$ of compatible size. If Assumption 1 is satisfied, such a function can always be constructed [21].

Remark 7 The accuracy of the enclosures constructed in this section depends on the choice of $v, V, s, S$, and $\Omega$. A thorough analysis of the conservatism of the ellipsoidal bounds as a function of these parameters goes beyond the scope of this paper. Methods to construct such functions can be found in other works [21, 34].

In the following, we introduce the functions

$$
\begin{aligned}
\Phi_{1}(Q, A) & =A Q+Q A^{\top} \\
\Phi_{2}(Q, W, \sigma) & =\sigma Q+\frac{1}{\sigma} W \\
\varphi_{3}\left(q_{1}, q_{2}, Q_{1}, Q_{2}, \kappa\right) & =\kappa Q_{1} Q_{2}^{-1}\left(q_{2}-q_{1}\right) \\
\Phi_{3}\left(q_{1}, q_{2}, Q_{1}, Q_{2}, \kappa\right) & =\kappa\left(I-\left\|q_{1}-q_{2}\right\|_{Q_{2}^{-1}}^{2} I-Q_{1} Q_{2}^{-1}\right) Q_{1}
\end{aligned}
$$

for all scalars $\kappa, \sigma$ as well as all vectors $q_{1}, q_{2}$ and matrices $A, B, W, Q, Q_{1}, Q_{2}$ with compatible dimensions. Similarly, we introduce the variables

$$
y=\left(q_{\mathrm{B}}, q_{\mathrm{F}}, Q_{\mathrm{B}}, Q_{\mathrm{F}}\right) \in \mathbb{Y}
$$

and

$$
\lambda=\left(A_{\mathrm{F}}, A_{\mathrm{B}}, B_{\mathrm{F}}, B_{\mathrm{B}}, \sigma_{\mathrm{B}}, \sigma_{\mathrm{F}}, \mu_{\mathrm{B}}, \mu_{\mathrm{F}}, \kappa_{\mathrm{B}}, \kappa_{\mathrm{F}}\right) \in \mathbb{L}
$$

together with the domains $\mathbb{Y}=\mathbb{R}^{n_{x}} \times \mathbb{R}^{n_{x}} \times \mathbb{S}_{++}^{n_{x}} \times \mathbb{S}_{++}^{n_{x}}$ and $\mathbb{L}=\mathbb{R}^{n_{x} \times n_{x}} \times$ $\mathbb{R}^{n_{x} \times n_{x}} \times \mathbb{R}^{n_{x} \times n_{u}} \times \mathbb{R}^{n_{x} \times n_{u}} \times \mathbb{R}_{+}^{6}$. The functions $F: \mathbb{Y} \times \mathbb{R}^{n_{u}} \times \mathbb{R}^{n_{x}} \times \mathbb{S}_{++}^{n_{x}} \times \mathbb{L} \rightarrow \mathbb{Y}$ with $F=\left(F_{1}, F_{2}, F_{3}, F_{4}\right)$ and

$$
\begin{aligned}
F_{1}\left(t, y, u_{1}, \lambda\right)= & f\left(q_{\mathrm{B}}, u_{1}, v\right)-\varphi_{3}\left(q_{\mathrm{B}}, s(t), Q_{\mathrm{B}}, S(t), \kappa_{\mathrm{B}}\right) \\
F_{2}\left(t, y, u_{1}, \lambda\right)= & f\left(q_{\mathrm{F}}, u_{1}, v\right)+\varphi_{3}\left(q_{\mathrm{F}}, q_{\mathrm{B}}, Q_{\mathrm{F}}, Q_{\mathrm{B}}, \kappa_{\mathrm{F}}\right) \\
F_{3}\left(t, y, u_{1}, \lambda\right)= & \Phi_{1}\left(Q_{\mathrm{B}}, A_{\mathrm{B}}\right)-\Phi_{2}\left(Q_{\mathrm{B}}, B V B^{\top}, \sigma_{\mathrm{B}},\right) \\
- & \Phi_{2}\left(Q_{\mathrm{B}}, \Omega\left(A_{\mathrm{B}}, B_{\mathrm{B}}, q_{\mathrm{B}}, u_{1}, v, Q_{\mathrm{B}}\right), \mu_{B}\right) \\
& -\Phi_{3}\left(q_{\mathrm{B}}, s(t), Q_{\mathrm{B}}, S(t), \kappa_{\mathrm{B}}\right) \\
F_{4}\left(t, y, u_{1}, \lambda\right)= & \Phi_{1}\left(Q_{\mathrm{F}}, A_{\mathrm{F}}\right)+\Phi_{2}\left(Q_{\mathrm{F}}, B V B^{\top}, \sigma_{\mathrm{F}}\right) \\
+ & \Phi_{2}\left(Q_{\mathrm{F}}, \Omega\left(A_{\mathrm{F}}, B_{\mathrm{F}}, q_{\mathrm{F}}, u_{1}, v, Q_{\mathrm{F}}\right), \mu_{\mathrm{F}}\right) \\
+ & \Phi_{3}\left(q_{\mathrm{F}}, q_{\mathrm{B}}, Q_{\mathrm{F}}, Q_{\mathrm{B}}, \kappa_{\mathrm{F}}\right)
\end{aligned}
$$

and $G: \mathbb{Y} \times \mathbb{Y} \rightarrow \mathbb{Y}$ given by

$$
\begin{aligned}
& G(y(0), y(T)) \\
& \quad=\left(q_{\mathrm{F}}(0)-x_{0}, q_{\mathrm{B}}(T)-s(T), Q_{\mathrm{F}}(0), Q_{\mathrm{B}}(T)-S(T)\right) .
\end{aligned}
$$

determine, respectively, the right-hand side and constraint function of the boundaryvalue problem that is needed in the following theorem. 
Theorem 3 Let Assumptions 1, 2, and 3 be satisfied and let the Lebesgue integrable function $u_{1}:[0, T] \rightarrow \mathbb{U}_{1}$ be given. Let $y: \mathbb{R} \rightarrow \mathbb{Y}$ and $\lambda: \mathbb{R} \rightarrow \mathbb{L}$ be any functions satisfying the boundary value problem

$$
\begin{aligned}
\forall t \in[0, T], \quad \dot{y}(t)=F\left(t, y(t), u_{1}(t), \lambda(t)\right) \\
0 \quad=G(y(0), y(T)) .
\end{aligned}
$$

Then, the set-valued functions

$$
Y_{\mathrm{B}}(t)=\mathcal{E}\left(q_{\mathrm{B}}(t), Q_{\mathrm{B}}(t)\right) \quad \text { and } \quad Y_{\mathrm{F}}(t)=\mathcal{E}\left(q_{\mathrm{F}}(t), Q_{\mathrm{F}}(t)\right)
$$

satisfy the conditions of Theorem 2 on $[0, T]$. That is, the set-valued function $Y_{\mathrm{F} \cap \mathrm{B}}: \mathbb{R} \rightarrow \mathbb{K}_{\mathrm{C}}^{n_{x}}$ with

$$
\forall t \in[0, T], \quad Y_{\mathrm{F} \cap \mathrm{B}}(t)=\mathcal{E}\left(q_{\mathrm{F}}(t), Q_{\mathrm{F}}(t)\right) \cap \mathcal{E}\left(q_{\mathrm{B}}(t), Q_{\mathrm{B}}(t)\right)
$$

is an enclosure of $X\left[u_{1}\right]$ on $[0, T]$.

A proof of Theorem 3 can be found in Appendix B.

\section{Tractable approximation of differential games}

We now present a conservative and tractable approximation of (6). This approximation is constructed by leveraging on the properties of the ellipsoidal approximation presented in the previous section. We assume that two bounding functions $H: \mathbb{R} \times \mathbb{R}^{n_{y}} \rightarrow \mathbb{R}^{n_{h}}$ and $M: \mathbb{R}^{n_{y}} \rightarrow \mathbb{R}^{n_{h}}$ satisfying

$$
\begin{aligned}
H(t, y) \leq 0 & \Longrightarrow \mathcal{E}\left(q_{\mathrm{F}}, Q_{\mathrm{F}}\right) \cap \mathcal{E}\left(q_{\mathrm{B}}, Q_{\mathrm{B}}\right) \subseteq \mathbb{X}_{1}(t) \\
M(y) \leq 0 & \Longrightarrow \mathcal{M}\left(\mathcal{E}\left(q_{\mathrm{F}}, Q_{\mathrm{F}}\right) \cap \mathcal{E}\left(q_{\mathrm{B}}, Q_{\mathrm{B}}\right)\right) \leq 0
\end{aligned}
$$

are available. Notice that the construction of these functions is akin to the construction of the nonlinearity bounder $\Omega$ [32]; see also Remark 7 .

Let us consider the optimal control problem

$$
\inf _{x, y,} \quad M(y(T)) \quad \text { s.t. }\left\{\begin{array}{l}
\forall t \in[0, T] \\
\dot{x}(t)=f\left(x(t), u_{1}(t), u_{2}(t)\right) \\
\dot{y}(t)=F\left(y(t), u_{1}(t), \lambda(t)\right) \\
u_{1}(t) \in \mathbb{U}_{1}, u_{2}(t) \in \mathbb{U}_{2} \\
y(t) \in \mathbb{Y}, \lambda(t) \in \mathbb{L} \\
x(t) \in \mathbb{X} u_{2}(t) \\
0 \geq H(t, y(t)) \\
0=G(y(0), y(T)) .
\end{array}\right.
$$

Theorem 3 implies that any feasible point of is a feasible point of 6 . The auxiliary state $x$ is used to enforce lower-level feasibility of the control $u_{1}$. 


\subsection{Numerical illustration}

We consider a differential game with dynamics given by

$$
\begin{array}{ll}
\forall t \in[0, T], & \dot{x}(t)=\left(\begin{array}{c}
x_{1}(t)+\frac{1}{2} x_{2}(t)+u_{1}(t), \\
\frac{3}{2} x_{1}(t)+x_{2}(t)+u_{2}(t)
\end{array}\right) \\
\text { and } & x(0)=(0,0)^{\top}
\end{array}
$$

with $T=\frac{3}{2}$. The path constraints are given by

$$
\mathbb{X}_{1}(t)=[-6,6]^{2} \quad \text { and } \quad \mathbb{X}_{2}(t)=R(t) \mathcal{E}(s, S)
$$

for all $t \in[0, T]$ with

$$
\begin{gathered}
s=\left(\begin{array}{c}
\frac{5}{50} \\
-\frac{71}{25}
\end{array}\right), \quad S=\left(\begin{array}{cc}
\frac{802}{25} & \frac{16}{5} \\
\frac{16}{5} & \frac{802}{25}
\end{array}\right), \\
\text { and } \quad R(t)=\left(\begin{array}{cc}
\frac{21}{20} \cos \left(\frac{\pi t}{2}\right) & -\frac{19}{20} \sin \left(\frac{\pi t}{2}\right) \\
\frac{21}{20} \sin \left(\frac{\pi t}{2}\right) & \frac{19}{20} \cos \left(\frac{\pi t}{2}\right)
\end{array}\right) .
\end{gathered}
$$

The control sets are $\mathbb{U}_{1}=[-1.4,0]$ and $\mathbb{U}_{2}=[0,2]$. The Mayer objective function is given by $m(x)=x_{1}-x_{2}$.

The bounding functions $M$ and $H$ can, in this example, be constructed without introducing further conservatism using the result from Proposition 1 in Appendix B. Problem 26) was formulated and solved numerically with ACADO Toolkit 19] using a multiple shooting discretization with 10 equidistant intervals, and a Runge-Kutta integrator of order $4 / 5$.

Figure 2 shows projections onto the $x_{1^{-}}$(top) and $x_{2}$-axis (bottom) planes of the ellipsoidal enclosures $Y_{\mathrm{B}}$ (light gray), the pointwise-in-time intersection of $Y_{\mathrm{B}}$ and $Y_{\mathrm{F}}$ (dark gray), and an inner approximation of $X\left[u_{1}^{\star}\right]$ (black) - computed by Monte Carlo simulation with $10^{4}$ trajectories.

The optimal value of $(26)$ is -1.59 . Recall that 26 is only a conservative approximation of (6), since Theorem 3 only provides a means to construct outer approximations of the exact forward reachable set of the game. However, the conservatism of the solution can be evaluated a posteriori by solving the optimal control problem of Player 2. Here, we find that Player 2 can at most achieve an optimal value of -3.67 . Thus, the optimal value of the exact differential game is overestimated by approximately 2.08. If one wishes to further reduce this overestimation, one would have to abandon the idea to work with ellipsoids and use more accurate set parameterizations. An in depth analysis of such general set parameterizations is, however, beyond the scope of this paper.

Last but not least, we also solve the above game for the case that Player 2 has no state constraints. In this case, the differential game reduces to a standard robust optimal control problem and the optimal value of Player 2 is 3.85. This illustrates the importance of taking state constraints in dynamic games into account and highlights the differences between robust optimal control and more general zero-sum differential games with state constraints. 

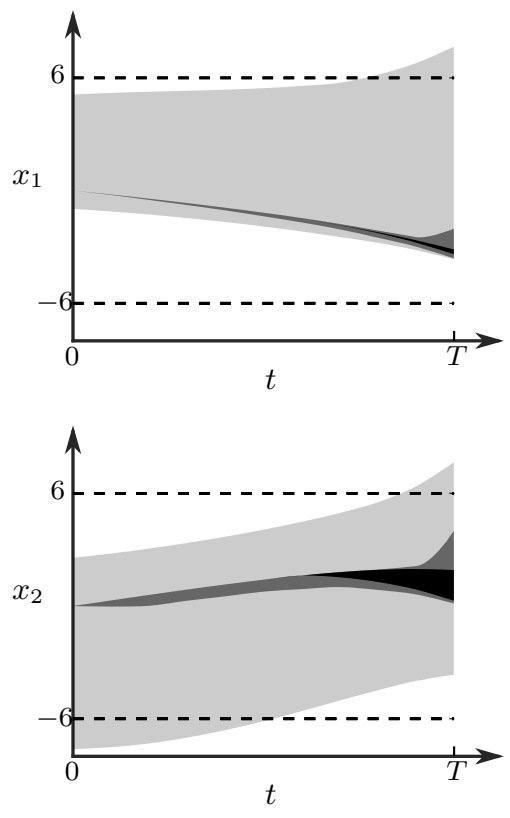

Figure 2: Projections of $Y_{\mathrm{B}}$ (light gray) and the pointwise-in-time intersection of $Y_{\mathrm{B}}$ and $Y_{\mathrm{F}}$ (dark gray) onto the $x_{1^{-}}$and $x_{2}$-axis. 


\section{Conclusion}

This paper has presented a set-theoretic framework for the numerical analysis of zero-sum differential games with state constraints. In particular, it introduced a novel backward-forward reachable set splitting scheme, which can be used by the first player to compute the reachable set of states of the second player. This splitting scheme was then used to derive convex outer approximations for the reachable set of the game using boundary value constrained differential inequalities. A particular emphasis was placed on ellipsoidal outer approximations, which lead to the conservative but tractable approximation (26) of the solution of the original Stackelberg differential game (6). The advantage of $(26)$ is that this is a standard optimal control problem, which can be solved using state-ofthe art optimal control solvers. The effectiveness of the approach was illustrated by means of a numerical example.

\section{Acknowledgements}

This research was supported by the National Natural Science Foundation China, Grant-No. 61473185; as well as ShanghaiTech University, Grant-No. F-0203-14012 .

\section{References}

[1] J.P. Aubin. Viability theory. Springer Science \& Business Media, 2009.

[2] M. Bardi and P. Soravia. Hamilton-Jacobi equations with singular boundary conditions on a free boundary and applications to differential games. Transactions of the American Mathematical Society, 325(1):205-229, 1991.

[3] T. Başar. A tutorial on dynamic and differential games. In Dynamic games and applications in economics, pages 1-25. Springer, 1986.

[4] T. Başar and P. Bernhard. H-infinity optimal control and related minimax design problems: a dynamic game approach. Springer Science \& Business Media, 2008.

[5] T. Basar and G.J. Olsder. Dynamic noncooperative game theory, volume 23. Siam, 1999.

[6] T. Basar and H. Selbuz. Closed-loop Stackelberg strategies with applications in the optimal control of multilevel systems. IEEE Transactions on Automatic Control, 24(2):166-179, 1979.

[7] A. Bensoussan. Stackelberg differential games. In Estimation and Control of Dynamical Systems, pages 493-521. Springer, 2018.

[8] F. Blanchini and S. Miani. Set-theoretic methods in control. Springer, 2008. 
[9] M.H. Breitner. The genesis of differential games in light of Isaacs contributions. Journal of Optimization Theory and Applications, 124(3):523-559, 2005 .

[10] A. Bressan and F.S. Priuli. Infinite horizon noncooperative differential games. Journal of Differential Equations, 227:230-257, 2006.

[11] P. Cardaliaguet, M. Quincampoix, and P. Saint-Pierre. Pursuit differential games with state constraints. SIAM Journal on Control and Optimization, 39(5):1615-1632, 2001.

[12] P. Cardaliaguet, M. Quincampoix, and P. Saint-Pierre. Differential games through viability theory: old and recent results. In S. Jorgensen, M. Quincampoix, and T.L. Vincent, editors, Advances in Dynamic Game Theory, volume 9, pages 3-35. Annals of the International Society of Dynamic Games, Birkhäuser Boston, 2007.

[13] M. Diehl, B. Houska, O. Stein, and P. Steuermann. A lifting method for generalized semi-infinite programs based on lower level Wolfe duality. Computational Optimization and Applications, 54:189-210, 2013.

[14] E.J. Dockner, S. Jorgensen, N. Van Long, and G. Sorger. Differential games in economics and management science. Cambridge University Press, 2000.

[15] M.B. Feldman. A proof of Lusin's theorem. American Math. Monthly, 88:191-192, 1981.

[16] A. Friedman. Differential Games. Wiley, New York, 1971.

[17] R. Hettich and K.O. Kortanek. Semi-infinite programming: theory, methods, and applications. SIAM review, 35(3):380-429, 1993.

[18] B. Houska. Robust optimization of dynamic systems. $\mathrm{PhD}$ thesis, Katholieke Universiteit Leuven, 2011.(ISBN: 978-94-6018-394-2), 2011.

[19] B. Houska, H.J. Ferreau, and M. Diehl. ACADO toolkit-An open-source framework for automatic control and dynamic optimization. Optimal Control Applications and Methods, 32(3):298-312, 2011.

[20] B. Houska, J.C. Li, and B. Chachuat. Towards rigorous robust optimal control via generalized high-order moment expansion. Optimal Control Applications and Methods, 39(2):489-502, 2018.

[21] B. Houska, F. Logist, J. Van Impe, and M. Diehl. Robust optimization of nonlinear dynamic systems with application to a jacketed tubular reactor. Journal of Process Control, 22(6):1152-1160, 2012.

[22] R. Isaacs. Differential games: a mathematical theory with applications to warfare and pursuit, control and optimization. John Wiley and Sons, New York, 1965. 
[23] H.T. Jongen, J.J. Rückmann, and O. Stein. Generalized semi-infinite optimization: a first order optimality condition and examples. Mathematical Programming, 83(1-3):145-158, 1998.

[24] N.N. Krasovskii and A.I. Subbotin. Game-Theoretical Control Problems. Springer-Verlag, Berlin, 1988.

[25] A.B. Kurzhanski and I. Vályi. Ellipsoidal calculus for estimation and control. Birkhäuser, 1997.

[26] W. Langson, I. Chryssochoos, S.V. Raković, and D.Q. Mayne. Robust model predictive control using tubes. Automatica, 40(1):125-133, 2004.

[27] J.E. Littlewood. Littlewood's Miscellany. Cambridge University Press, 1986. Edited by Bollobas, B.

[28] J. Nash. Non-cooperative games. Annals of Mathematics, 2:286-295, 1951.

[29] G. Papavassilopoulos and J. Cruz. Nonclassical control problems and Stackelberg games. IEEE Transactions on Automatic Control, 24(2):155-166, 1979 .

[30] H.J. Pesch, M. Plail, and D Munich. The maximum principle of optimal control: a history of ingenious ideas and missed opportunities. Control and Cybernetics, 38(4A):973-995, 2009.

[31] O. Stein. How to solve a semi-infinite optimization problem. European Journal of Operational Research, 223(2):312-320, 2012.

[32] M.E. Villanueva, B. Chachuat, and B. Houska. Robust optimal feedback control for periodic biochemical processes. In Proceedings of the 10th IFAC International Symposium on Advanced Control of Chemical Processes, China, pages 750-755, 2018.

[33] M.E. Villanueva, B. Houska, and B. Chachuat. Unified framework for the propagation of continuous-time enclosures for parametric nonlinear ODEs. Journal of Global Optimization, 62(3):575-613, 2015.

[34] M.E. Villanueva, R. Quirynen, M. Diehl, B. Chachuat, and B. Houska. Robust MPC via min-max differential inequalities. Automatica, 77:311$321,2017$.

[35] H. Von Stackelberg. The Theory of the Market Economy. Oxford University Press, 1952.

\section{A Proof of Theorem 2}

The proof of Theorem 2 is non-trivial and, therefore, it has been divided into different sub-sections, which build upon each other. 


\section{A.1 Technical preliminaries}

Let us consider a general differential equation of the form

$$
\dot{x}(t)=g(x(t), w(t)) \quad \text { with } \quad x(0)=x_{0}
$$

with $g$ being Lipschitz continuous in $x$ and continuous in the external input $w:[0, T] \rightarrow \mathbb{W} \subseteq \mathbb{K}^{n_{w}}$. Let

$$
X_{\mathbb{T}}(t)=\left\{\begin{array}{l|l}
\xi \in \mathbb{R}^{n_{x}} & \begin{array}{l}
\exists x \in \mathbb{W}_{1,1}^{n_{x}}, \exists w \in \mathbb{T}^{n_{w}}: \\
\forall \tau \in[0, t] \\
\dot{x}(\tau)=g(x(\tau), w(\tau)) \\
x(0)=x_{0} \\
w(\tau) \in \mathbb{W}, x(t)=\xi
\end{array}
\end{array}\right\}
$$

denote the reachable sets of this differential equation for a given subset $\mathbb{T} \subseteq \mathbb{L}_{1}$. Let $\mathbb{C}^{n_{w}} \subseteq \mathbb{L}_{1}^{n_{w}}$ denote the set of bounded continuous functions. Now, a direct consequence of Lusin's theorem [15] and Gronwall's lemma is that

$$
\forall t \in \mathbb{R}, \quad \operatorname{cl}\left(X_{\mathbb{C}}(t)\right)=\operatorname{cl}\left(X_{\mathbb{L}_{1}}(t)\right)
$$

In other words, if we are only interested in the closure of a reachable set, we may simply replace Lebesgue integrable functions by continuous functions in a statement without altering its conclusion. In the following, we will use such replacements without saying this explicitly at all places. In particular, we assume, without loss of generality, that $u_{1}$ is any given continuous function.

\section{A.2 Constrained set propagation operators}

Let

$$
\Pi\left(t_{2}, t_{1}, X_{1}, Z\right)=\left\{\begin{array}{l|l}
\xi \in \mathbb{R}^{n_{x}} & \begin{array}{l}
\exists x \in \mathbb{W}_{1,1}^{n_{x}}, \exists u_{2} \in \mathbb{L}_{1}^{n_{u}}: \\
\forall t \in\left[t_{1}, t_{2}\right], \\
\dot{x}(t)=f\left(x(t), u_{1}(t), u_{2}(t)\right) \\
x\left(t_{1}\right) \in X_{1}, x(t) \in Z(t), \\
u_{2}(t) \in \mathbb{U}_{2}, x\left(t_{2}\right)=\xi
\end{array}
\end{array}\right\},
$$

denote the constrained set propagation operator of (1), which is defined for all $X_{1} \in \mathbb{K}_{\mathrm{C}}^{n_{x}}$, all $Z:\left[t_{1}, t_{2}\right] \rightarrow \mathbb{K}_{\mathrm{C}}^{n_{x}}$, and all $t_{1}, t_{2} \in \mathbb{R}$ with $t_{1} \leq t_{2}$. Moreover, let

$$
\pi(\xi, X)=\underset{\xi^{\prime} \in X}{\operatorname{argmin}}\left\|\xi-\xi^{\prime}\right\|_{2}^{2} .
$$

denote the Euclidean projection of a point $\xi \in \mathbb{R}^{n_{x}}$ onto a compact convex set $X \in \mathbb{K}_{\mathrm{C}}^{n_{x}}$. Now, the key idea is to introduce the auxiliary differential equation

$$
\forall t \in[0, T], \quad \dot{z}(t)=f_{K}\left(t, z(t), u_{2}(t), Z(t)\right)
$$


with

$$
f_{K}\left(t, \xi, \nu_{2}, X\right)=-K(\xi-\pi(\xi, X))+f\left(\xi, u_{1}(t), \nu_{2}\right)
$$

for all $\xi \in \mathbb{R}^{n_{x}}, \nu_{2} \in \mathbb{R}^{n_{u}}$, and all $X \in \mathbb{K}_{\mathrm{C}}^{n_{x}}$. Here, $K>0$ is a tuning parameter that can be interpreted as a proportional control gain of an additional control term, which can be used to steer 28 towards $X$ whenever $z(t)$ is outside of $X$. Let

$$
\Pi_{K}\left(t_{2}, t_{1}, X_{1}, Z\right)=\left\{\begin{array}{l|l}
\xi \in \mathbb{R}^{n_{x}} & \begin{array}{l}
\exists z \in \mathbb{W}_{1,1}^{n_{x}}, \exists u_{2} \in \mathbb{L}_{1}^{n_{u}}: \\
\forall t \in\left[t_{1}, t_{2}\right], \\
\dot{z}(t)=f_{K}\left(t, z(t), u_{2}(t), Z(t)\right) \\
x\left(t_{1}\right) \in X_{1}, u_{2}(t) \in \mathbb{U}_{2} \\
x\left(t_{2}\right)=\xi
\end{array}
\end{array}\right\},
$$

denote the set-propagation operator of $[28$. In analogy to the propagation operator $\Pi, \Pi_{K}$ is defined for all $X_{1} \in \mathbb{K}_{\mathrm{C}}^{n_{x}}$, all $Z:\left[t_{1}, t_{2}\right] \rightarrow \mathbb{K}_{\mathrm{C}}^{n_{x}}$, and all $t_{1}, t_{2} \in \mathbb{R}$ with $t_{1} \leq t_{2}$.

Lemma 1 Let Assumptions 1 and 2 be satisfied. Let $Y, Z:[0, T] \rightarrow \mathbb{K}_{\mathrm{C}}^{n_{x}}$ be any given set valued function such that the intersection $Y(t) \cap Z(t)$ is, for all $t \in[0, T]$, nonempty and such that the functions $V[Y(\cdot) \cap Z(\cdot)](c)$ and $V[Y(\cdot)](c)$ are, for all $c \in \mathbb{R}^{n_{x}}$, Lipschitz continuous on $[0, T]$. If the differential inequality

$$
\begin{aligned}
& \dot{V}[Y(t)](c) \geq \max _{\xi, \nu_{2}} c^{\boldsymbol{\top}} f_{K}\left(t, \xi, \nu_{2}, Y(t) \cap Z(t)\right) \\
& \text { s.t. }\left\{\begin{array}{r}
c^{\top} \xi=V[Y(t)](c) \\
\xi \in Y(t) \\
\nu_{2} \in \mathbb{U}_{2}
\end{array}\right. \\
& V[Y(0)](c) \geq V\left[X_{0}\right](c)
\end{aligned}
$$

is satisfied for all $c \in \mathbb{R}^{n_{x}}$ and all $t \in[0, T]$ for a given initial set $X_{0} \in \mathbb{K}_{\mathrm{C}}^{n_{x}}$, then

$$
\forall t \in[0, T], \quad \Pi\left(0, t, X_{0}, Z\right) \subseteq \Pi_{K}\left(0, t, X_{0}, Z\right) \subseteq Y(t) .
$$

Proof. First, notice that $\pi$ satisfies $\pi(\xi, Y(t) \cap Z(t))=\xi$ whenever $\xi \in$ $Y(t) \cap Z(t)$. Thus, we have

$$
f_{K}\left(t, \xi, u_{2}(t), Y(t) \cap Z(t)\right)=f\left(\xi, u_{1}(t), u_{2}(t)\right)
$$

for all $\xi \in Y(t) \cap Z(t)$. This implies the first inclusion,

$$
\forall t \in[0, T], \quad \Pi\left(0, t, X_{0}, Z\right) \subseteq \Pi_{K}\left(0, t, X_{0}, Z\right) .
$$

In order to establish the remaining inclusion, we assume for a moment that $u_{2}$ is constant. Since the set $Y(t) \cap Z(t)$ is nonempty and convex, $\pi(\cdot, Y(t) \cap Z(t))$ is non-expansive. Thus, this function is uniformly Lipschitz continuous on $[0, T] \times$ $\mathbb{U}_{2}$ with Lipschitz constant 1 . Furthermore, by the Lipschitz continuity of the function $V[Y(\cdot) \cap Z(\cdot)](c)$ the function $\pi(\xi, Y(\cdot) \cap Z(\cdot))$ is continuous. This, 
together with Assumption 1, implies that $f_{K}$ is jointly continuous in $\left(t, \xi, \nu_{2}\right)$ as well as Lipschitz continuous in $\xi$, uniformly on $[0, T] \times \mathbb{U}_{2}$.

At this point, it is important to notice that $Y$ and $Z$ are arbitrary but given, thus the right-hand side function $f_{K}$ is only a function of time, the state and the parameter $u_{2}$. We can now replicate the arguments of all the steps in the proof of Theorem 3 in 33 to 28 - observing that we have established the required properties of $f_{K}$-to obtain the second inclusion

$$
\forall t \in[0, T], \quad \Pi_{K}\left(0, t, X_{0}, Z\right) \subseteq Y(t) .
$$

The assumption that $u_{2}$ is constant can be removed using the same argument as in [33, Remark 2].

Corollary 1 Let the conditions of Lemma 1 hold. Assume, in addition, that $Y(t) \cap \operatorname{int}(Z(t)) \neq \varnothing$, for all $t \in[0, T]$. If $Y$ is such that the differential inequality

$$
\begin{aligned}
& \dot{V}[Y(t)](c) \geq \max _{\xi, \nu_{2}} c^{\top} f\left(\xi, u_{1}(t), \nu_{2}\right) \\
& \text { s.t. } \quad\left\{\begin{array}{c}
c^{\top} \xi=V[Y(t)](c) \\
\xi \in Y(t) \cap \operatorname{int}(Z(t)) \\
\nu_{2} \in \mathbb{U}_{2}
\end{array}\right. \\
& V[Y(0)](c) \geq V\left[X_{0}\right](c)
\end{aligned}
$$

is satisfied for all $c \in \mathbb{R}^{n_{x}}$ and all $t \in[0, T]$, then

$$
\forall t \in[0, T], \quad \Pi\left(0, t, X_{0}, Z\right) \subseteq Y(t) .
$$

Proof. The proof proceeds in two steps. First, we show that the result holds under the stronger assumption that $Y$ is such that $Y(t)$ is strictly convex for all $t \in[0, T]$ and that it satisfies the differential inequality

$$
\begin{gathered}
\dot{V}[Y(t)](c) \geq \max _{\xi, \nu_{2}} c^{\top} f\left(\xi, u_{1}(t), \nu_{2}\right) \\
\text { s.t. }\left\{\begin{aligned}
c^{\top} \xi & =V[Y(t)](c) \\
\xi & \in Y(t) \cap Z(t) \\
\nu_{2} & \in \mathbb{U}_{2}
\end{aligned}\right.
\end{gathered}
$$

for all $t \in[0, T]$ and all $c \in \mathbb{R}^{n_{x}}$.

Let the set

$$
F[Y(t)](c)=\left\{\begin{array}{l|l}
\xi \in \mathbb{R}^{n_{x}} \mid \begin{array}{c}
c^{\top} \xi=V[Y(t)](c) \\
\xi \in Y(t)
\end{array}
\end{array}\right\} .
$$

be the supporting facet of $Y(t)$ in the direction $c \in \mathbb{R}^{n_{x}}$. Notice that for any given pair $(t, c) \in[0, T] \times \mathbb{R}^{n_{x}}$, the set $F[Y(t)](c)$ is a singleton. Thus, for any given pair $(t, c) \in[0, T] \times \mathbb{R}^{n_{x}}$, there are only two possible cases: 
Case 1: The set $F[Y(t)](c) \cap Z(t)$ is nonempty. In this case, we have

$$
F[Y(t)](c) \cap Z(t) \subseteq Y(t) \cap Z(t) .
$$

Since $f$ and $f_{K}$ coincide on $Y(t) \cap Z(t)$ they also coincide on $F[Y(t)](c) \cap Z(t)$. Thus, $Y$ and $Z$ satisfy the differential inequality 29 from Lemma 1 at $(t, c)$ for any $K>0$.

Case 2: The set $F[Y(t)](c) \cap Z(t)$ is empty. This is only possible, if

$$
\max _{\xi \in F[Y(t)](c)} c^{\top} \xi>\max _{\xi^{\prime} \in Y(t) \cap Z(t)} c^{\top} \xi^{\prime} .
$$

Thus, it follows that

$$
\forall \xi \in F[Y(t)](c), \quad c^{\top}(\xi-\pi(\xi, Y(t) \cap Z(t))>0,
$$

since $\pi(\xi, Y(t) \cap Z(t)) \in Y(t) \cap Z(t)$. Therefore, the term

$$
\begin{aligned}
& c^{\top} f_{K}\left(t, \xi, u_{2}(t), Y(t) \cap Z(t)\right) \\
& =-K \underbrace{c^{\top}(\xi-\pi(\xi, Y(t) \cap Z(t)))}_{>0}+c^{\top} f\left(\xi, u_{1}(t), u_{2}(t)\right)
\end{aligned}
$$

can be made arbitrarily small by choosing a sufficiently large $K$.

Thus, we have shown that there exists for every pair $(t, c) \in[0, T] \times \mathbb{R}^{n_{x}}$ a sufficiently large $K$, such that $Y$ and $Z$ satisfy the differential inequality (29) from Lemma 1. In particular $Y$ and $Z$ satisfy the (strengthened) conditions from Lemma 1 in the limit as $K \rightarrow \infty$. We must mention that one should be careful when taking this limit, as the Lipschitz constant of $f_{K}$ diverges for $K \rightarrow \infty$. Fortunately, one can apply the following topological argument: if $Y$ and $Z$ satisfy the above hypothesis, one can always construct enclosures $Y_{\epsilon}$ and $Z_{\epsilon}$ satisfying the conditions of Lemma 1 for a sufficiently large $K$ and such that the Hausdorff distance between $Y_{\epsilon}(t)$ and $Y(t)$ (as well as $Z_{\epsilon}(t)$ and $Z(t)$ ) converges to zero as $\epsilon \rightarrow 0$ - uniformly on $[0, T]$. This claim follows readily from our continuity assumptions. As the images of these functions are compact, one can pass to the topological closure to show that Lemma 1 implies that

$$
\forall t \in[0, T], \quad \Pi\left(0, t, X_{0}, Z\right) \subseteq Y(t),
$$

if (31) holds.

At this point, we construct an enclosure $Y_{\epsilon}$ of the operator $\Pi$, such that $Y_{\epsilon}(t)$ is, for all $t \in[0, T]$, compact, and strictly convex. Then, we apply the procedure above and a continuity argument to show that (31) also holds in the limit as $\epsilon \rightarrow 0$, for set-valued functions with convex and compact images. The technical proof for this claim is analogous to Step S2 in the proof of Thm. 3 in [33.

Finally, observe that the only difference between (31) and the condition of Corollary 1 and is that in the latter, the intersection $Y(t) \cap Z(t)$ has been replaced by the tighter set $Y(t) \cap \operatorname{int}(Z(t))$. However, as $Z(t)$ has a nonempty interior, we have

$$
\operatorname{cl}(Y(t) \cap \operatorname{int}(Z(t)))=\operatorname{cl}(Y(t) \cap Z(t)),
$$


i.e., the statement of the corollary is not affected if we replace $Z(t)$ in the intersection by its interior. This follows from the fact that the supremum of a continuous function over any bounded set in $\mathbb{R}^{n_{x}}$ coincides with the maximum of the function over the closure of this set. Thus, we conclude that the statement of the corollary holds.

\section{A.3 Complete Proof of Theorem 2}

The statement of Theorem 2 can be obtained by a repeated application of Corollary 1. First, the corollary is applied to the reversed differential equation

$$
\dot{z}(t)=-f\left(z(t), u_{1}(T-t), u_{2}(t)\right) \text { with } z(0) \in \mathbb{X}_{2}(T)
$$

with $Y(t)=Y_{\mathrm{B}}(T-t), Z(t)=\mathbb{X}_{2}(T-t)$, and $X_{0}=\mathbb{X}_{2}(T)$. Reversing time once more, shows that the inequalities for $\dot{V}\left[Y_{\mathrm{B}}\right](c)$ and $V\left[Y_{\mathrm{B}}(t)\right](c)$ in Theorem 2 imply the inclusion $X_{\mathrm{B}}\left[u_{1}\right](t) \subseteq Y_{\mathrm{B}}(t)$, which is valid for all $t \in[0, T]$.

Now, we apply Corollary 1 directly with $Y=Y_{\mathrm{F}}, Z=Y_{\mathrm{B}}$ and $X_{0}=\left\{x_{0}\right\}$. This yield the inequalities for $V\left[Y_{\mathrm{F}}\right](c)$ and $V\left[Y_{\mathrm{F}}(t)\right](c)$ in Theorem 2 implying that $Y_{\mathrm{F}}$ is an enclosure for $X_{\mathrm{F}}\left[u_{1}\right]$ on $[0, T]$. Since $X_{\mathrm{B}}\left[u_{1}\right]$ is, by definition, also an enclosure for $X_{\mathrm{F}}\left[u_{1}\right]$ on $[0, T]$, it follows that the inclusion $Y_{\mathrm{F}}(t) \cap Y_{\mathrm{B}}(t) \supseteq$ $X_{\mathrm{F}}\left[u_{1}\right](t)$ holds for all $t \in[0, T]$, yielding the statement of the theorem.

\section{B Proof of Theorem 3}

This appendix is divided into a number of subsections which build upon each other and lead to the proof of Theorem 3 . Moreover, we use the technical convention from Section A.1.

\section{B.1 Support functions of set propagation operators}

We recall that the constrained set-propagation operator $\Pi$ has been introduced in (27), see Appendix A, while the shorthand $\Gamma$ is defined in (23).

Lemma 2 Let Assumptions 1 and 2 be satisfied. Let $Y, Z:[0, T] \rightarrow \mathbb{K}_{C}^{n_{x}}$ be any set-valued functions, such that $\bar{Y}(t)$ and $Y(t) \cap Z(t)$ are, for all $t \in[0, T]$, strictly convex and the intersection is nonempty; and such that $V[Y(\cdot)](c)$ and $V[Y(\cdot) \cap Z(\cdot)](c)$ are, for all $c \in \mathbb{R}^{n_{x}}$, differentiable. If there exists a continuous function $\alpha: \mathbb{R} \rightarrow \mathbb{R}$ with $\alpha(0)=0$ such that the inequality

$$
V[\Pi(t, t+h, Y(t), Z)](c) \leq V[Y(t+h)](c)+h \alpha(h),
$$

holds for all $c \in \mathbb{R}^{n_{x}}$ with $\|c\| \leq 1$ and all $t \in[0, T]$, then

$$
\dot{V}[Y(t)](c) \geq V\left[\Gamma\left(u_{1}(t), c, Y(t), Z(t)\right)\right](c)
$$

holds for all $c \in \mathbb{R}^{n_{x}}$ with $\|c\| \leq 1$ and all $t \in[0, T]$. 
Proof. We first show that the set-propagation operator $\Pi$ satisfies, for all $c \in \mathbb{R}^{n_{x}}$, the differential inequality

$$
\begin{array}{r}
\dot{V}[\Pi(t, t+h, Y(t), Z)](c) \\
\geq V\left[\Gamma\left(u_{1}(t), c, Y(t), Z(t)\right)\right](c) .
\end{array}
$$

The proof of this statement is indirect. Let $c \in \mathbb{R}^{n_{x}}$ be a vector for which 32 does not hold. Then, there exists a point

$$
\xi^{\star} \in \underset{x \in Y(t)}{\operatorname{argmax}} c^{\boldsymbol{\top}} x \quad \text { with } \quad \xi^{\star} \in \operatorname{int}(Z(t)) .
$$

Otherwise we have $V\left[\Gamma\left(u_{1}(t), c, Y(t), Z(t)\right)\right](c)=-\infty$, and the inequality 32 holds. Now, it follows from the definition of $\Gamma\left(u_{1}(t), c, Y(t), Z(t)\right)$ that there exists a $\nu_{2}^{\star} \in \mathbb{U}_{2}$ such that

$$
V\left[\Gamma\left(u_{1}(t), c, Y(t), Z(t)\right)\right](c)=c^{\top} f\left(\xi^{\star}, u_{1}(t), \nu_{2}^{\star}\right),
$$

i.e., we have

$$
\dot{V}[\Pi(t, t+h, Y(t), Z)](c)<c^{\top} f\left(\xi^{\star}, u_{1}(t), \nu_{2}^{\star}\right) .
$$

Since $\xi^{\star} \in \operatorname{int}(Z(t))$, the inequality contradicts the definition of $\Pi$. Thus, 32 must hold for all $c \in \mathbb{R}^{n_{x}}$.

This means that there exists a continuous function $\beta: \mathbb{R} \rightarrow \mathbb{R}$ such that

$$
\begin{aligned}
V[\Pi(t, t+h, Y(t), Z)](c)-V[Y(t)](c) \\
\quad \geq h V\left[\Gamma\left(u_{1}(t), c, Y(t), Z(t)\right)\right](c)-h \beta(h) .
\end{aligned}
$$

Thus, using the assumptions of this lemma, we can conclude that

$$
\begin{aligned}
& V[Y(t+h)](c)-V[Y(t)](c) \\
& \quad \geq V[\Pi(h, Y(t), Z)](c)-V[Y(t)](c)-h \alpha(h) \\
& \quad \geq h V\left[\Gamma\left(u_{1}(t), c, Y(t), Z(t)\right)\right](c)-h[\alpha(h)+\beta(h)] .
\end{aligned}
$$

Dividing the last inequality by $h$ on both sides and taking the limit for $h \rightarrow 0$, we obtain the statement of the lemma.

\section{B.2 Ellipsoidal calculus}

The following proposition summarizes two known results from the field of ellipsoidal calculus.

Proposition 1 Let $q_{1}, q_{2} \in \mathbb{R}^{n_{x}}$ and $Q_{1}, Q_{2} \in \mathbb{S}_{++}^{n_{x}}$ be given.

1. If $\lambda \in(0,1)$, then

$$
\mathcal{E}\left(q_{1}, Q_{1}\right) \oplus \mathcal{E}\left(q_{2}, Q_{2}\right) \subseteq \mathcal{E}\left(q_{1}+q_{2}, \frac{Q_{1}}{\lambda}+\frac{Q_{2}}{1-\lambda}\right) .
$$


2. If $\kappa=\left(\kappa_{1}, \kappa_{2}\right) \in \mathbb{R}_{+}^{2}$ satisfies

$$
\begin{gathered}
1=\kappa_{1}\left(1-q_{1}^{\top} Q_{1}^{-1} q_{1}\right)+\kappa_{2}\left(1-q_{2}^{\top} Q_{2}^{-1} q_{2}\right) \\
+q(\kappa)^{\top} Q(\kappa) q(\kappa),
\end{gathered}
$$

with $Q(\kappa) \in \mathbb{S}_{++}^{n_{x}}$ and $q(\kappa) \in \mathbb{R}^{n_{x}}$ given by

$$
\begin{aligned}
\tilde{Q}(\kappa) & =\left(\kappa_{1} Q_{1}^{-1}+\kappa_{2} Q_{2}^{-1}\right)^{-1} \\
\tilde{q}(\kappa) & =Q_{N}(\kappa)\left(\kappa_{1} Q_{1}^{-1} q_{1}+\kappa_{2} Q_{2}^{-1} q_{2}\right),
\end{aligned}
$$

then $\mathcal{E}\left(q_{1}, Q_{1}\right) \cap \mathcal{E}\left(q_{2}, Q_{2}\right) \subseteq \mathcal{E}(\tilde{q}(\kappa), \tilde{Q}(\kappa))$.

Proof. The proofs for these two statements can be found in [25. See also [18 for alternative derivations.

Corollary 2 Let Assumptions 1, 2, and 3 be satisfied and let $r: \mathbb{R} \rightarrow \mathbb{R}^{n_{x}}$ and $R: \mathbb{R} \rightarrow \mathbb{S}_{++}^{n_{x}}$ be given differentiable functions. Let $q: \mathbb{R} \rightarrow \mathbb{R}^{n_{x}}$ and $Q: \mathbb{R} \rightarrow \mathbb{S}_{++}^{n_{x}}$ be differentiable and satisfy the differential equations

$$
\begin{aligned}
\dot{q}(t)= & f(q(t), u(t), v)+\varphi_{3}(q(t), r(t), Q(t), R(t), \kappa(t)) \\
\dot{Q}(t)= & \Phi_{1}(Q(t), A(t))+\Phi_{2}\left(Q(t), B(t) V B(t)^{\top}, \sigma(t)\right) \\
& +\Phi_{2}\left(Q(t), \Omega\left(A(t), B(t), q(t), u_{1}(t), v, Q(t)\right), \mu(t)\right) \\
& +\Phi_{3}(q(t), r(t), Q(t), R(t), \kappa(t)),
\end{aligned}
$$

on the interval $[0, T]$, for any given functions $A: \mathbb{R} \rightarrow \mathbb{R}^{n_{x} \times n_{x}}, B: \mathbb{R} \rightarrow$ $\mathbb{R}^{n_{x} \times n_{u}}, \sigma, \mu: \mathbb{R} \rightarrow \mathbb{R}_{++}$and $\kappa: \mathbb{R} \rightarrow \mathbb{R}_{+}$. Then, the ellipsoidal set valued function $Y$ with $Y(t)=\mathcal{E}(q(t), Q(t))$ satisfies, for all $c \in \mathbb{R}^{n_{x}}$, all $t \in[0, T]$, and any $Z: \mathbb{R} \rightarrow \mathbb{K}_{\mathrm{C}}^{n_{x}}$ with $Z(t) \subseteq \mathcal{E}(r(t), R(t))$ the differential inequality

$$
\begin{aligned}
\dot{V}[Y(t)](c) & \geq V\left[\Gamma\left(u_{1}(t), c, Y(t), Z(t)\right)\right](c) \\
V[Y(t)](c) & \geq V\left[X_{0}\right](c)
\end{aligned}
$$

with $X_{0}=\mathcal{E}(q(0), Q(0))$.

Proof. This proof relies on the application of Lemma 2 with $Y(t)=$ $\mathcal{E}(q(t), Q(t))$. Notice that by differentiability of $q$ and $Q$, the function $V[\mathcal{E}(q((\cdot), Q(\cdot))](c)$ is also differentiable on $[0, T]$, for all $c \in \mathbb{R}^{n_{x}}$. Let

$$
\begin{aligned}
\tilde{x}(t, h)= & x(t)+h f\left(x(t), u_{1}(t), u_{2}(t)\right) \\
= & x(t)+h f\left(q(t), u_{1}(t), v\right)+h A(t)(x(t)-q(t)) \\
& +h B(t)\left(u_{2}(t)-v\right)+h n(t)
\end{aligned}
$$

denote an Euler approximation of the original ODE at time $t$, where

$$
n(t) \in \mathcal{E}\left(0, \Omega\left(A(t), B(t), q(t), u_{1}(t), v(t), Q(t)\right)\right)
$$


and $x(t) \in \mathcal{E}(q(t), Q(t))$. The second statement of Proposition 1 implies that by setting

$$
\begin{aligned}
\tilde{Q}(t, h) & =\left(\kappa_{1}(t, h) Q(t)^{-1}+\kappa_{2}(t, h) R(t)^{-1}\right)^{-1} \\
\tilde{q}(t, h) & =\left(\kappa_{1}(t, h) Q(t)^{-1} q(t)+\kappa_{2}(t, h) R(t)^{-1} r(t)\right) \tilde{Q}(t, h) .
\end{aligned}
$$

we have, by our assumption $Z(t) \subseteq \mathcal{E}(r(t), R(t))$,

$$
Y(t) \cap Z(t) \subseteq \mathcal{E}(\tilde{q}(t, h), \tilde{Q}(t, h))
$$

as long as $\kappa_{1}(t, h), \kappa_{2}(t, h) \geq 0$ satisfy

$$
\begin{aligned}
\tilde{q}(t, h)^{\top} \tilde{Q}(t, h) \tilde{q}(t, h)= & 1-\kappa_{1}(t, h)\left(1-q(t)^{\top} Q(t)^{-1} q(t)\right) \\
& -\kappa_{2}(t+h)\left(1-r(t)^{\top} R(t)^{-1} r(t)\right) .
\end{aligned}
$$

Moreover, since $\mathbb{U}_{2} \subseteq \mathcal{E}(v, V)$, a repeated application of the first statement in Proposition 1 shows that setting

$$
\begin{aligned}
\hat{Q}(t, h)= & \frac{1}{\lambda_{1}(t, h)}(I+h A(t)) \tilde{Q}(t, h)(I+h A(t))^{\top} \\
& +\frac{h^{2}}{\lambda_{2}(t, h)} B(t) V B(t)^{\top} \\
& \left.+\frac{h^{2}}{\lambda_{3}(t, h)} \Omega\left(A(t), B(t), q(t), u_{1}(t), v, Q(t)\right)\right) \\
\hat{q}(t, h)= & q_{N}(t+h)+h f\left(q_{N}(t+h), u_{1}(t), v\right),
\end{aligned}
$$

for any function $\lambda_{1}(t, h), \lambda_{2}(t, h), \lambda_{3}(t, h)>0$ with $\lambda_{1}(t, h)+\lambda_{2}(t, h)+\lambda_{3}(t, h)=$ 1 , implies

$$
\begin{aligned}
& V[\Pi(t+h, t, Y(t), Z)](c) \\
& \quad \leq V[\mathcal{E}(\hat{q}(t, h), \hat{Q}(t, h))](c)+h \gamma(h)
\end{aligned}
$$

for a continuous function $\gamma: \mathbb{R} \rightarrow \mathbb{R}$ with $\gamma(0)=0$, for all $c$ with $\|c\|=1$, because the Euler discretization is accurate for $h \rightarrow 0$. Next, we substitute

$$
\begin{aligned}
& \lambda_{1}(t, h)=1-h \sigma(t)-h \mu(t) \\
& \lambda_{2}(t, h)=h \sigma(t) \\
& \lambda_{3}(t, h)=h \mu(t) \\
& \kappa_{1}(t, h)=1-h \kappa(t),
\end{aligned}
$$

while $\kappa_{2}(t, h)$ is defined implicitly by (34). Differentiating the above formulas 
for $\hat{q}$ and $\hat{Q}$ with respect to $h$ yields

$$
\begin{aligned}
\frac{\mathrm{d}}{\mathrm{d} h} \hat{q}(t, 0)= & f\left(\hat{q}(t, 0), u_{1}(t), v\right) \\
& +\varphi_{3}(\hat{q}(t, 0), r(t), \hat{Q}(t, 0), R(t), \kappa(t)) \\
\frac{\mathrm{d}}{\mathrm{d} h} \hat{Q}(t, 0)= & \Phi_{1}(\hat{Q}(t, h), A(t)) \\
+ & \Phi_{2}\left(\hat{Q}(t, 0), B(t) V B(t)^{\top}, \sigma(t)\right) \\
+ & \Phi_{2}(\hat{Q}(t, 0), \Omega(A(t), B(t), \widetilde{q}(t, t), \\
& \left.\left.u_{1}(t), v, \hat{Q}(t, 0)\right), \mu(t)\right) \\
& +\Phi_{3}(\hat{q}(t, 0), r(t), \hat{Q}(t, 0), R(t), \kappa(t)) .
\end{aligned}
$$

Since the right-hand of these derivatives coincide with the differential equations for $q$ and $Q$, we must have

$$
q(t+h)=\hat{q}(t, h)+O\left(h^{2}\right) \text { and } Q(t, h)=\hat{Q}(t, 0)+O\left(h^{2}\right),
$$

i.e., 35 implies that there exists a continuous function $\alpha: \mathbb{R} \rightarrow \mathbb{R}$ with $\alpha(0)=0$ and

$$
\begin{aligned}
& V[\Pi(t+h, t, Y(t), Z)](c) \\
& \quad \leq V[\mathcal{E}(q(t+h), Q(t+h))](c)+h \alpha(h) .
\end{aligned}
$$

Thus, the statement of this corollary turns into an immediate of consequence of Lemma 2 .

\section{B.3 Complete Proof of Theorem 3}

The statement of Theorem 3 follows by applying the result of Corollary 2 twice. Firstly, we apply the corollary to the reverse dynamic system

$$
\frac{\partial}{\partial t} z(t)=-f\left(z(t), u_{1}(T-t), u_{2}(t)\right) \text { with } z(0) \in \mathbb{X}_{2}(T) .
$$

with $q=q_{\mathrm{B}}, Q=Q_{\mathrm{B}}, r=s, R=S$ and $q_{\mathrm{B}}(0)=s(T)$ and $Q_{\mathrm{B}}(0)=S(T)$. This yields an enclosure for the backward tube $X_{\mathrm{B}}\left[u_{1}\right]$. And secondly, we apply Corollary 2 to (11) with $q=q_{\mathrm{F}}, Q=Q_{\mathrm{F}}, r=q_{\mathrm{B}}, R=Q_{\mathrm{B}}, q_{\mathrm{F}}(0)=x_{0}$, and $Q_{\mathrm{F}}(0)=0$, which yields the enclosure of the forward tube $X_{\mathrm{F}}\left[u_{1}\right]$. Since both $Y_{\mathrm{B}}(\cdot)=\mathcal{E}\left(q_{\mathrm{B}}(\cdot), Q_{\mathrm{B}}(\cdot)\right)$ and $Y_{\mathrm{F}}(\cdot)=\mathcal{E}\left(q_{\mathrm{F}}(\cdot), Q_{\mathrm{F}}(\cdot)\right)$ are enclosures of $X\left[u_{1}\right]$ on $[0, T]$, constructing a set-valued function $Y_{\mathrm{F} \cap \mathrm{B}}$ by taking their pointwise-in-time intersection yields the statement of the theorem. 